\title{
EXAMPLES IN THE THEORY OF THE SCHUR GROUP
}

\author{
BY CHARLES FORD AND GERALD JANUSZ
}

Communicated by Joseph J. Rotman, April 23, 1973

Let $K$ be a subfield of a cyclotomic extension of the rational field $Q$. The Schur group of $K$ is the subgroup $S(K)$ of the Brauer group of $K$ consisting of those classes of central simple $K$ algebras represented by an algebra which appears as a direct summand of a group algebra $Q[G]$ for some finite group $G$. For a prime $p$ let $S(K)_{p}$ denote the subgroup consisting of elements having $p$-power order. It is known by [1] that $S(K)_{p}$ can have an element of order $p^{a}$ only when a primitive $p^{a}$ root of unity, $\varepsilon_{p^{a}}$, is in $K$.

Suppose $K$ is a field which satisfies $Q\left(\varepsilon_{p^{a}}\right) \subseteq K \subseteq Q\left(\varepsilon_{n}\right)$ and $p^{a}$ is the highest power of $p$ dividing $n$. It is known that

$$
S(K)_{p}=K \otimes S\left(Q\left(\varepsilon_{p^{a}}\right)\right)_{p}
$$

in the case $K=Q\left(\varepsilon_{n}\right)$. That is every element in $S(K)_{p}$ is represented by an algebra $K \otimes B$ with $B$ central simple over $Q\left(\varepsilon_{p^{a}}\right)[2]$.

The assertion (1) also holds for $K$ if $p$ does not divide $\left(Q\left(\varepsilon_{n}\right): K\right)$. In this paper we present, for each prime $p$, fields $K$ for which (1) does not hold.

Let $p$ be a prime and $r$ and $s$ distinct primes such that $r \equiv s \equiv 1 \bmod p$. Then the field $L=Q\left(\varepsilon_{p}, \varepsilon_{r}, \varepsilon_{s}\right)$ has two nontrivial automorphisms $\sigma, \tau$ which satisfy

(i) $\sigma^{p}=\tau^{p}=1$

(ii) $\sigma$ fixes $\varepsilon_{p}$ and $\varepsilon_{r} ; \tau$ fixes $\varepsilon_{p}$ and $\varepsilon_{s}$.

Let $K$ be the subfield of $L$ fixed by $\langle\sigma, \tau\rangle$. Let $A$ be the algebra defined by

$$
\begin{gathered}
A=\sum L u_{\sigma}^{i} u_{\tau}^{j} ; \\
u_{\sigma}^{p}=u_{\tau}^{p}=1, \quad u_{\sigma} u_{\tau}=\varepsilon_{p} u_{\tau} u_{\sigma} ; \\
u_{\sigma} x=\sigma(x) u_{\sigma}, \quad u_{\tau} x=\tau(x) u_{\tau} \text { for } x \text { in } L .
\end{gathered}
$$

Then $A$ is central simple over $K$ and is a simple component of the group algebra $Q[G]$ where $G$ is the group of order $p^{3} r s$ generated by $u_{\sigma}, u_{\tau}, \varepsilon_{p r s}$. We use this algebra for several examples.

Let $f_{r}$ be the exponent of $r$ mod $s$; that is, $f_{r}$ is the least positive integer $f$ such that $r^{f} \equiv 1 \bmod s$. Similarly let $f_{s}$ be the exponent of $s \bmod r$.

THEOREM. (1) If $p \mid f_{r}$ then the $r$-local index of $A$ is $p$. In particular $A$ has index $p$ if either $p \mid f_{r}$ or $p \mid f_{s}$.

(2) If $A$ has $r$-local index $p$ and $p^{2}$ divides either $r-1$ or $f_{r}$ then $A$ is not

AMS (MOS) subject classifications (1970). Primary 16A26; Secondary16A40, $20 \mathrm{C05.}$ 
similar to $K \otimes B$ for any $Q\left(\varepsilon_{p}\right)$-central simple algebra $B$ in $S\left(Q\left(\varepsilon_{p}\right)\right)$. In particular, $S(K)_{p} \neq K \otimes S\left(Q\left(\varepsilon_{p}\right)\right)_{p}$.

We remark that when $p^{2}$ does not divide either $r-1$ or $s-1$ then $A$ is similar to $K \otimes B$ with $B$ representing a class in $S\left(Q\left(\varepsilon_{p}\right)\right)$. In fact $B$ can be explicitly described as follows. Let the Galois group of $Q\left(\varepsilon_{p}, \varepsilon_{r}, \varepsilon_{s}\right)=L$ over $Q\left(\varepsilon_{p}\right)$ be $\langle\alpha, \beta\rangle$ where $\alpha$ has order $r-1$ and fixes $\varepsilon_{s}$ while $\beta$ has order $s-1$ and fixes $\varepsilon_{r}$. Then

$$
\begin{gathered}
B=\sum L u_{\alpha}^{i} u_{\beta}^{j} ; \\
u_{\alpha}^{r-1}=u_{\beta}^{s-1}=1, \quad u_{\alpha} u_{\beta}=\varepsilon u_{\beta} u_{\alpha} ; \\
u_{\alpha} x=\alpha(x) u_{\alpha}, \quad u_{\beta} x=\beta(x) u_{\beta} \quad \text { for } x \in L .
\end{gathered}
$$

Here $\varepsilon$ is a suitable power of $\varepsilon_{p}$.

It should be observed also that for any prime $p$, there exist primes $r, s$ which satisfy the conditions in (2) of the theorem. In fact a little more can be said. Let $p$ be any prime and $m$ a positive integer. By Dirichlet's theorem there exist infinitely many primes $r$ which satisfy $r \equiv 1 \bmod p^{m}$. Now for any such $r$ there exist infinitely many primes $s$ such that $s \equiv 1 \bmod p^{m}$ and the exponent of $s \bmod r$ equals $p^{m}$. In fact the Dirichlet density of the set of such $s$ is $1 /(r-1)$.

One specific case where condition (2) holds occurs with $p=3, r=7$, $s=37$. Then $f_{r}=9$ and $f_{s}=3$.

Suppose we construct the algebra $A$ as above using $p, r, s$ and $m \geqq 2$ which satisfy the divisibility conditions just above. Let $p^{b}$ and $p^{c}$ be the highest power of $p$ dividing $r-1$ and $s-1$ respectively. Suppose $p^{d}$ is the highest power of $p$ dividing $f_{r}$ and $p^{m}=f_{s}$. Notice $b, c \geqq m$. Then $p^{b+d}$ and $p^{c+m}$ are the exact powers of $p$ dividing $r^{f_{r}}-1$ and $s^{f_{s}}-1$ respectively. The algebra $A$ has index $p$ and we ask for which values of $n$ will $K\left(\varepsilon_{p^{n}}\right)$ be a splitting field for $A$ ? In case $d=0$ the least $n$ for which $K\left(\varepsilon_{p^{n}}\right)$ splits $A$ is $n=c+m$. In case $d \neq 0$ then the least $n$ is the larger of the numbers $b+d$ and $c+m$. In any case the least $n$ is larger than $m$.

We formulate this more abstractly as follows.

THEOREM. Given a prime $p$ and an integer $m \geqq 2$ there exists a finite group $G$ and a simple direct summand $A$ of $Q[G]$ having center $K$ and index $p$ such that

(i) $\varepsilon_{p} \in K, \varepsilon_{p^{2}} \notin K$,

(ii) for some integer $n>m, K\left(\varepsilon_{p^{n}}\right)$ is a splitting field for A but no proper subfield is a splitting field.

By the general theory of algebras we know $A$ has a splitting field $E$ such that $(E: K)=p$. Here $\left(K\left(\varepsilon_{p^{n}}\right): K\right)=p^{n-1}$ can be made as large as desired by selecting suitable $G$ and yet $K\left(\varepsilon_{p^{n}}\right)$ is a "minimal splitting field" in the sense that no proper subfield splits the algebra. 


\section{REFERENCES}

1. M. Benard and M. Schacher, The Schur subgroup. II, J. Algebra 22 (1972), 378-385.

2. G. J. Janusz, The Schur group of cyclotomic fields, J. Number Theory (to appear).

Department of Mathematics, Washington University, St. Louis, Missouri 63130

Department of Mathematics, University of Illinois, Urbana, IlLinois 61801 